\title{
Local study of the corrosion kinetics of hardened Portland cement under acid attack
}

\author{
Héloïse Gay, Thomas Meynet, Jean Colombani \\ Institut Lumière Matière; Université de Lyon; Université Claude Bernard Lyon 1; \\ CNRS, UMR 5306; Domaine scientifique de la Doua, F-69622 Villeurbanne cedex, \\ France
}

\begin{abstract}
Whereas the chemical reactions occurring during the acid corrosion of cementitious materials are now well known, their reaction rate have still not been measured. We propose here a methodology using digital holographic interferometry to access to the pure surface reaction rate. This method makes possible the differentiation between the dissolution and precipitation steps. We use it to measure the reaction rate constant of the dissolution of hardened Portland cement in aqueous solutions of nitric, sulfuric and hydrochloric acid at $\mathrm{pH} 2$. This quantity is seen to be unexpectedly similar for the three acids, with a value of the order of $1 \mathrm{mg} / \mathrm{m}^{2} / \mathrm{s}$. We have measured the evolution of this reaction rate constant with the $\mathrm{pH}$ in nitric acid. We have also measured the real $\mathrm{pH}$ of the solution at the material surface, which is always alkaline $(\mathrm{pH}>11)$, even for attacks by solution with $\mathrm{pH}$ as low as 1.
\end{abstract}

Keywords: A: reaction, A: kinetics, A: pH, C: corrosion, acid

\section{Introduction}

The extension of the durability of concrete buildings necessitates the understanding of its limiting factors, like creep, crack propagation by water freezing, degradation due to aggressive environments, etc. Among chemical attacks reducing the service life of construction materials, acid attack is particularly detrimental to materials as alkaline as cementitious materials.

Email address: Jean.Colombani@univ-lyon1.fr (Jean Colombani) 
Indeed the hydration products of cement are neutralized by protons, inducing dissolution and precipitation of new salts, destroying the microstructure of this hydraulic binder. The mechanisms of action of acid reagents on concrete, mainly originating outdoor in air pollution (dry deposition) and acid rains (wet deposition), have been investigated for a few decades, and the basic features are now identified $[1,2,3]$. These modes of action have also been studied indoor in the case of industrial constructions involving acidic environments $[4,5]$. These mechanisms, including heterogeneous reactions, ion substitution, mass loss, diffusion of solution in pores, strain, porosity evolution, leaching, etc. are complex, interact with each other, and lead to a loss of strength and stiffness of the material.

The investigation of acid attack on cement-based materials has been performed either with field measurements in urban areas $[6,7]$, or with laboratory measurements. The latter have used either simulated acid rain solutions $[7,8,9]$, or concentrated acid aqueous solutions [10], in order to accelerate the reactions. Sometimes, different reagents are tested simultaneously or alternatively $[10,4]$. In the course of these studies, three parameters are generally evaluated: the chemical composition of the runoff solution, the change of mass or size of the samples, and their strength decrease. Due to the complexity of the phenomenon, a quantity is generally not measured, although being the fundamental parameter of chemical kinetics: the reaction rate.

Up to now, models of degradation of concrete under acid attack use empirical dissolution laws [11]. We think that the knowledge of well-defined reaction parameters may help in the elaboration of the predicting tools of acid corrosion. As the reaction of dissolution is always combined with other phenomena like precipitation, flow of the solution, diffusion of dissolved species, etc. in standard acid attack setups, these experiments are not usable for pure corrosion rate measurement. Therefore we propose in this article an original methodology, using holographic interferometry, to access to the reaction rate of cementitious materials in acid solutions. We provide values of the pure dissolution rate of hardened Portland cement in three strong acids - nitric, sulfuric and hydrochloric acids - at various $\mathrm{pH}$ as first examples of measurements.

Our investigation of hydrated cement acid attack follows a non-conventional procedure, focusing on one single step of the phenomenon, namely on the first one, dissolution. Accordingly a comparison of our results with standard studies on the resistance of cement-based materials to acid is not directly possible [12]. Indeed these experiments are global ones, including dissolution, precipitation, cracking, volume expansion ... and do not enable to 


\begin{tabular}{cccccccc}
$\mathrm{CaO}$ & $\mathrm{SiO}_{2}$ & $\mathrm{Al}_{2} \mathrm{O}_{3}$ & $\mathrm{Fe}_{2} \mathrm{O}_{3}$ & $\mathrm{Na}_{2} \mathrm{O}$ & $\mathrm{MgO}$ & $\mathrm{K}_{2} \mathrm{O}$ & $\mathrm{SO}_{2}$ \\
\hline 62.7 & 17.7 & 5.5 & 4.1 & 0.4 & 0.8 & 0.7 & 3.7
\end{tabular}

Table 1: Composition (mass \%) of the ordinary Portland cement used in the corrosion experiments.

47

isolate pure dissolution. But we hope that our main results - values of the reaction rates, real value of the $\mathrm{pH}$ at the attacked surface, evidence of the tiny influence of the nature of the strong acid - will (i) find verification in standard studies, and (ii) help to model more accurately acid degradation of cementitious materials.

\section{Materials and method}

\subsection{Samples and acidic solutions}

We have used in this study ordinary Portland cement (CEM-1), the composition of which is detailed in table 1. It is made of at least $95 \%$ clinker. The cement paste was cast in Petri dishes and cut, after 28 days of curing in a moist environment, in order to obtain parallelepipedic samples of approximate dimensions $5 \times 5 \times 0.5 \mathrm{~mm}^{3}$. The water over cement ratio was 0.4 . The nitric, sulfuric and hydrochloric acid solutions were diluted with pure water to obtain the required $\mathrm{pH}$. These three inorganic acids being strong, the $\mathrm{pH}$ is the convenient quantity to indicate the content of aggressive species in solution. All experiments have been carried out at ambient temperature.

\subsection{Holographic interferometry}

Our final aim is the measurement of a reliable reaction rate of hardened cement in acid solutions. To be unquestionable, the experiments must guarantee that no other phenomenon (convection, precipitation ...) disturbs the measurement, to provide a pure dissolution rate. This implies that, during the acid attack of the samples, we are able (i) to observe the interface where the reaction proceeds, to study the reaction precisely where it occurs, and (ii) to access to the concentration field in the liquid soaking the solid, in order to detect all possible mass transport phenomena (diffusion, convection) and remove their contribution from the reaction rate. To achieve these two goals, we have carried out real-time digital holographic interferometry measurements. 



Figure 1: Photograph and diagram of the experimental cell, containing the acid solution and the hardened cement sample at the bottom.

Here we take advantage of the potentiality of holography to record phase objects, i.e., transparent objects exhibiting only variation of their index of refraction in our case. Here the investigated transparent object is the solution where the solid dissolves, contained in a transparent cell of dimensions $10 \times 10 \times 40 \mathrm{~mm}^{3}$ (figure 1 ). We record first the state of this corrosive liquid in a so-called reference digital hologram at time $t_{0}$. Here, we choose for $t_{0}$ the moment just before the solid is introduced in the cell. Then the hardened cement thin sample is placed at the bottom of the cell. Subsequently we record periodically the digital hologram of the liquid during the attack of the solid. Afterwards we perform numerically a superposition of the holograms at time $t_{0}$ and at the various times $t$ to obtain interferograms of the evolution of the state of the liquid between times $t_{0}$ and $t$ (figure 2). The evolution of the concentration $c$ in the solution, due to the ion release stemming from the attack, induces a change of the refractive index, hence of the phase of the liquid, which is visualized through interference fringes. We want to emphasize that holographic interferometry differentiates from classical interferometry in the fact that no external reference is needed, the object interfering virtually with a memory of itself [13].

A numerical procedure enables to reconstruct the two-dimensional concentration field in the liquid from the fringes (figure 3). As only concentration changes inside the cell here, and the concentration was initially uniform, interference fringes are iso-concentration curves. The fringes have always been observed horizontal, so we can deduce that concentration is horizontally invariant, and the evolution is only one-dimensional, in the vertical direction.

The chemical reaction is considered first-order, with the dissolution flux at the interface writing $J=k s_{\mathrm{r}}(1-c / s)$ with $k$ the reaction rate constant, $s_{\mathrm{r}}$ the reactive surface area, $c$ the concentration of the dissolved species and 

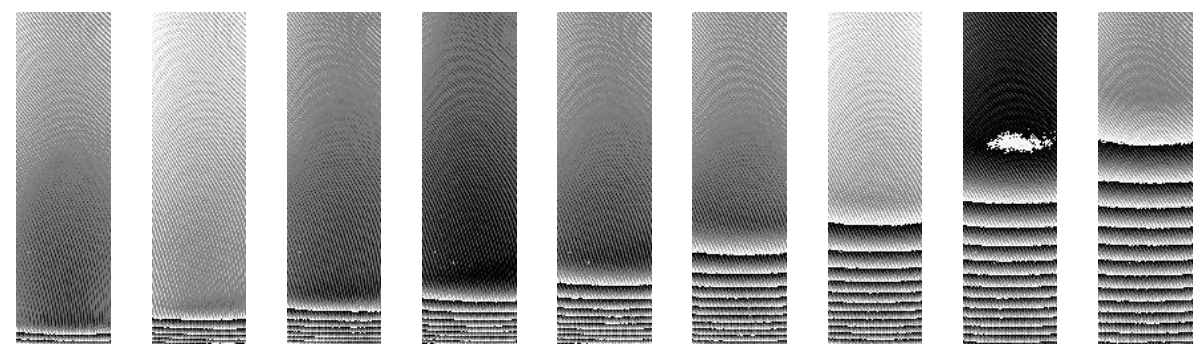

Figure 2: Digital holographic interferograms of the dissolution of a hardened Portland cement sample at the bottom of a nitric acid solution of $\mathrm{pH} 1.5$, at 5, 15, 30, 50, 80, 130, 220, 400 and 600 min after the introduction of the sample at the bottom of the cell. The dimension of each interferogram is $5 \times 18 \mathrm{~mm}^{2}$.

$s$ their solubility limit. The two physico-chemical quantities $s$ ans $k$ both depend on the thermodynamic conditions, the sole varying parameter of which here is the $\mathrm{pH}$. The reaction rate $J$ can be viewed as the velocity with which the concentration curve at the interface $z=0$ shifts upward (figure $3)$.

The solution of Fick's second law with this chemical reaction at the solidliquid interface, in a semi-infinite one-dimensional approximation, brings the theoretical concentration evolution $c(z, t)$ with vertical dimension $z$ and time $t$ in the cell:

$$
\begin{aligned}
c(z, t)=s\left[\operatorname{erfc}\left(\frac{z}{2 \sqrt{D t}}\right)-\exp \left[\frac{k\left(s_{\mathrm{r}} / s_{\mathrm{d}}\right) z}{D \rho s}+\left(\frac{k\left(s_{\mathrm{r}} / s_{\mathrm{d}}\right)}{D \rho s}\right)^{2} D t\right]\right. \\
\left.\times \operatorname{erfc}\left[\frac{z}{2 \sqrt{D t}}+\frac{k\left(s_{\mathrm{r}} / s_{\mathrm{d}}\right)}{D \rho s} \sqrt{D t}\right]\right]
\end{aligned}
$$

with $s_{\mathrm{d}}$ the section of the experimental cell, $\rho$ the density of the solution, and $D$ the diffusion coefficient of the dissolved species. The best fit of the experimental curves deduced from the holographic results with the analytic expression of $c(z, t)$ brings the pure surface reaction rate constant $k$, the solubility of the dissolved species $s$, and their mean diffusion coefficient $D$ (figure 3).

We stress on the fact that the solubility $s$ measured here is the saturation concentration of the dissolving components of hardened cement (see section 3 for the nature of these components). This solubility has no link with the components precipitating later in the course of the experiment.

Details on the experimental device and data analysis have been given elsewhere, applied to the situation of gypsum dissolution in water [14] and 


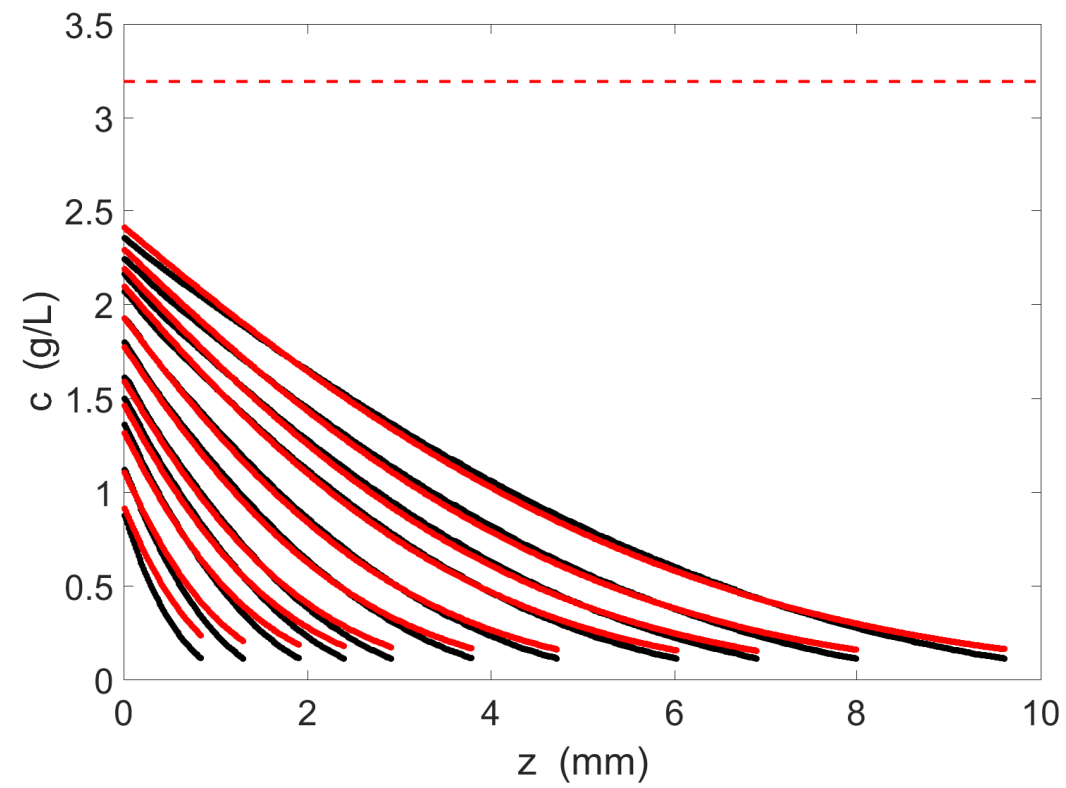

Figure 3: Experimental (black curves) and theoretical (red curves) concentration of dissolved cement hydrate in a nitric acid solution of $\mathrm{pH} 1.5$ versus vertical dimension. The curves correspond to the times $9,15,25,35,47,70,100,150,190,250$ and 350 min of the experiment. The horizontal line is the extrapolated saturation concentration $s$ of the dissolved species. The sample lies at $z=0$.

in various aqueous solutions [15].

This method of investigation of hydrated cement corrosion by acid solutions brings the following advantages:

1. The observation of the two-dimensional concentration field in the liquid enables to state that the mass transport of the dissolved species in our quiescent liquid is exclusively diffusional, thereby validating the use of Fick's law to determine the theoretical concentration profile. Indeed, the presence of any non-diffusive fluxes (natural convection, gravitational instability ...) would have induced a distortion of the interference fringes [16].

2. After a first step of pure dissolution, precipitation of salts stemming from the neutralization of the mineral occurs [1]. The pure dissolution rate can be estimated from the concentration curves only before this recrystallisation sets in. The holographic interferometry offers 


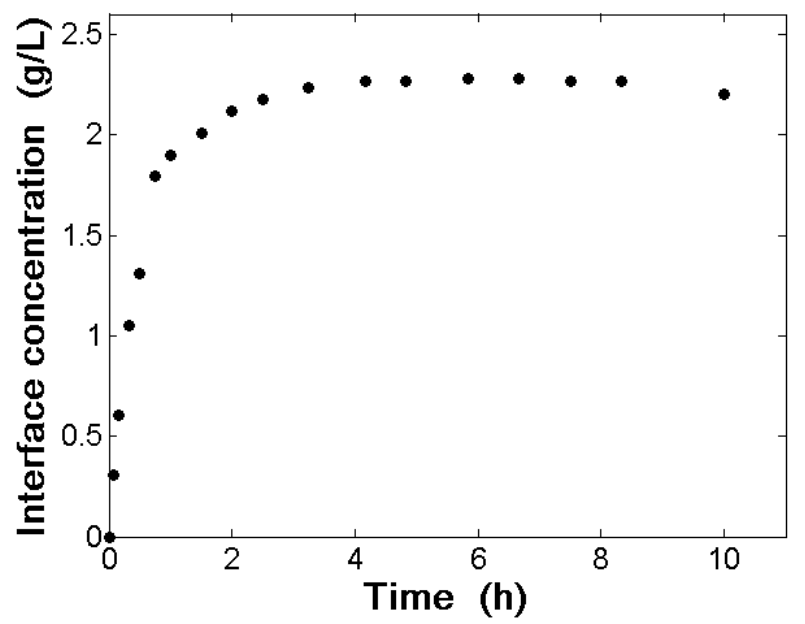

Figure 4: Evolution with time of the concentration of dissolved hardened cement at the solid-liquid interface $z=0$ during the dissolution of a hardened Portland cement sample at the bottom of a nitric acid solution of $\mathrm{pH} 1.5$.

the chance to follow the concentration at the solid-liquid interface $c(z=0, t)$. In figure 3 , this value can be read at the ordinate axis. It evolves upward due to the matter release caused by the attack. Precipitation consumes ions and thereby induces a decrease of the interface concentration. Therefore, as soon as $c(z=0, t)$ decreases, the corresponding concentration curves are excluded from the fitting, to assure that only dissolution is taken into account. An example of concentration evolution with time at the interface during dissolution is shown in figure 4 . In this case, the curves have been taken into account for the computation of the reaction rate constant only before the beginning of the plateau, at time $t \simeq 4 \mathrm{~h}$.

3. The good agreement between the experimental curves and the theoretical law constitutes a validation of our assumption that what occurs is a pure dissolution phenomenon with a first-order reaction.

\subsection{Refractive index of the solution}

The holographic interferometry device provides a two-dimensional phase map $\phi(y, z)$. The transformation of the phase into concentration has two requirements. First, the concentration must be known in each interferogram 


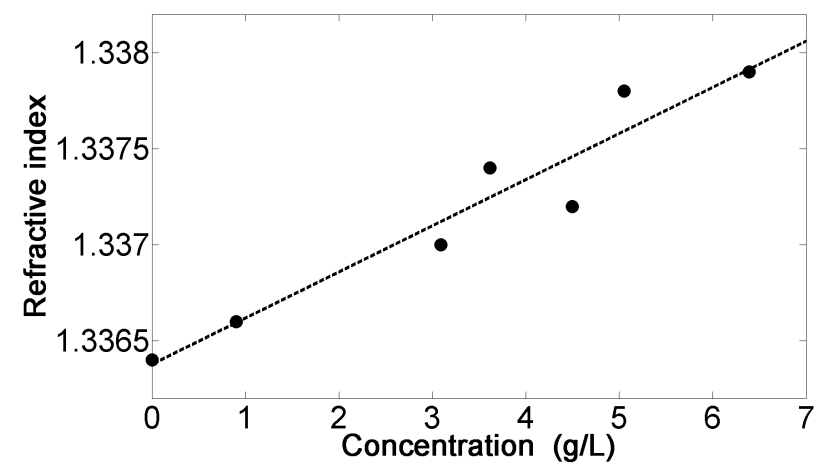

Figure 5: Index of refraction of an aqueous solution of nitric acid of $\mathrm{pH} 1$ vs concentration of dissolved hardened Portland cement. The line is a linear fit.

at least in one point, the rest of the absolute concentration field being deduced by comparison with this point. As the initial liquid is the pure acid aqueous solution, free from dissolved matter, the concentration at the top of the cell is always $c=0$. This is always true because, during the course of our experiments, we pay attention to the fact that no fringe has the time to reach the top of the cell. Secondly, the concentration is computed from the phase $\phi$ via the formula $c=\lambda \phi /[2 \pi e(\partial n / \partial c)]$, where $\lambda$ is the laser wavelength $(\lambda=532 \mathrm{~nm}), e$ the depth of the cell $(e=10 \mathrm{~mm})$ and $n$ the refractive index of the solution. The variation of the index of refraction of the solution with the concentration of dissolved species $\partial n / \partial c$ is therefore a crucial parameter. To gain this value, we have dissolved various quantities precisely weighed of finely ground hardened cement in pure aqueous solution of nitric acid of $\mathrm{pH} 1$. Then we have measured the refractive index of this solution with an Abbe refractometer (figure 5). The slope of the $n(c)$ curve is the searched parameter $\partial n / \partial c=2.4 \times 10^{-4} \mathrm{~L} / \mathrm{g}$.

We have measured the reaction rate of hydrated cement under acid attack at different $\mathrm{pH}$. So to estimate the influence of $\mathrm{pH}$ on $\partial n / \partial c$, the refractive index of an aqueous solution of pure nitric acid for various $\mathrm{pH}$ has been measured with the Abbe refractometer. It can be seen in figure 6 that the index of refraction is constant, except for very small $\mathrm{pH}$, where it increases of $0.2 \%$. In these conditions, we have estimated that the influence of the $\mathrm{pH}$ on $\partial n / \partial c$ is negligible. 




Figure 6: Index of refraction vs $\mathrm{pH}$ of an aqueous solution of nitric acid.

\section{Reactions during acid attack}

Sulfuric, hydrochloric and nitric acids have been chosen because they are ones of the main acidic components in acid rains [6]. The chemical reactions of hydrated cement in these acid solutions have already been studied in detail. As our aim is not to discuss these reactions but to measure their kinetics, we will just recall them briefly. Under acid $\mathrm{pH}$, all the constituents (calcium hydroxides, calcium silicate hydrates (CSH), calcium aluminate hydrates $(\mathrm{CAH}) \ldots$ ) dissolve at various velocities, and new salts precipitate. The first and faster dissolving component is calcium hydroxide -also called Portlandite- along the neutralization reactions [7]:

$$
\mathrm{Ca}(\mathrm{OH})_{2}+2 \mathrm{H}^{+} \longrightarrow \mathrm{Ca}^{2+}+2 \mathrm{H}_{2} \mathrm{O}
$$

The nature of the calcium salts crystallizing subsequently depend on the anion provided by the acid, typically gypsum and ettringite in the case of sulfuric acid. The severity of the attack is considered to be mainly dependent on the solubility of the precipitated salts. Indeed in the case of highly insoluble salts, e.g. fluoride salts, a dense layer forms at the interface, protecting the material [2]. But to try to understand more clearly the mechanisms at the beginning of the attack, we have restricted ourselves in this study to the first stage of the acid attack, where the main phenomenon is the dissolution of Portlandite, and we have not studied the subsequent precipitation of possibly protecting layers. Therefore we access here to the pure severity of the acid attack, whatever the further reactions. 


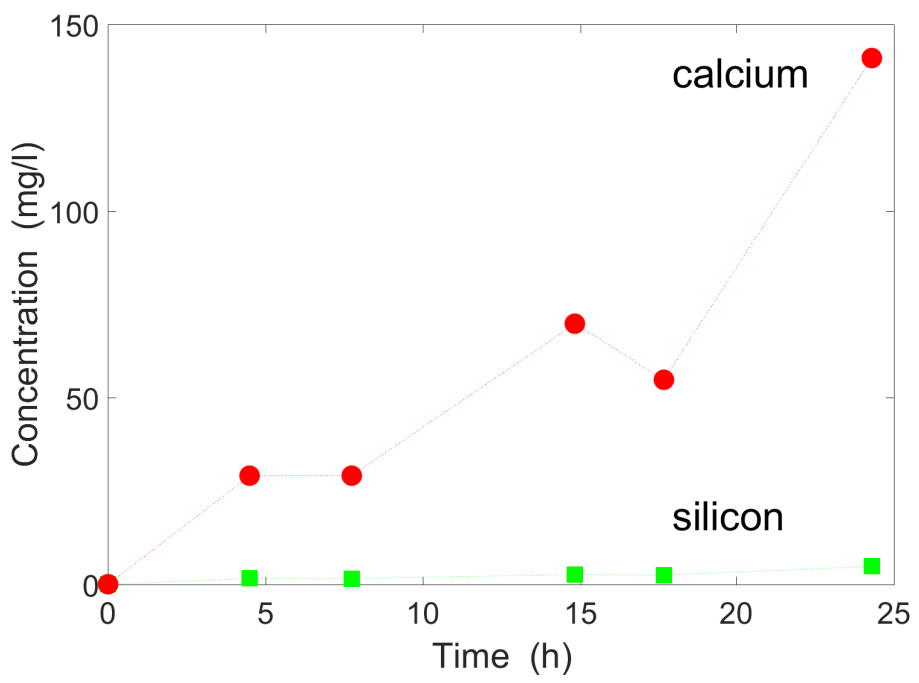

Figure 7: Concentration of dissolved calcium (red circles) and silicon (green squares) as a function of time during the attack of hardened Portland cement by quiescent nitric acid at $\mathrm{pH}=2$, measured with ICPAES.

To assess that the reaction scenario is really the above-mentioned one, we have performed additional chemical analyses of the attacked surface and of the soaking solution. The surface measurements have been carried out with the energy dispersive X-ray spectrometer (EDS) of a scanning electron microscope (SEM) and have brought the post mortem concentration of the main elements ( $\mathrm{Si}, \mathrm{Ca}, \mathrm{Al}, \mathrm{Na}, \mathrm{Mg}, \mathrm{K}, \mathrm{Ti}, \mathrm{Mn}, \mathrm{Fe}$ ) at the hardened cement surface after various attack durations. The solution measurements have been achieved with induced coupled plasma atomic emission spectroscopy (ICPAES) and enabled to access to the silicon, aluminum and calcium concentrations in the solvent after some attack durations. The solution has been stirred before sampling to guarantee the homogeneity of the concentrations.

The evolution with time of the calcium and silicon concentration in the attacking solution during an experiment is shown in figure 7. Besides, the time change of the concentration of calcium and silicon at the surface of the attacked hardened cement during the same experiment are presented in figure 8. The calcium concentration is seen to decrease at the solid surface and to increase in the liquid. Oppositely, the silicon concentration increases slowly at the surface and remains almost zero in the solution. These four 


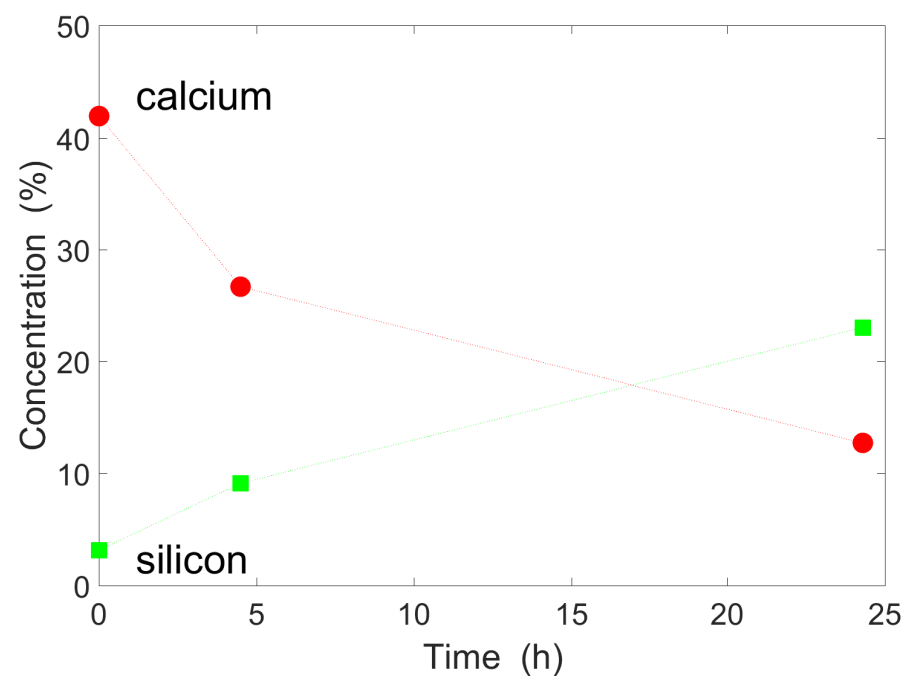

Figure 8: Concentration of calcium and silicon atoms (in percentage of total elements) $v s$ time at the surface of the attacked hardened cement of figure 7 , measured with SEM EDS.

features are in perfect agreement with the above-explained expected attack mechanism. The fastest dissolving component is Portlandite, so calcium atoms progressively disappear from the surface and calcium ions are released in the acid solution. On the other side, CSH dissolves orders of magnitude slower than Portlandite and the release of silicon ions in the solution is almost negligible during the course of our experiment. As surface Portlandite fade away, CSH is uncovered and the concentration of silicon at the very surface increases.

Except aluminum, the concentration of all other elements at the attacked surface is $1 \%$ or below during the experiment. The surface concentration of aluminum is $5 \%$ and remains constant during the attack duration. The concentration of aluminum in the solution during the experiment is always about $1 \mathrm{mg} / \mathrm{l}$, close to the measurement uncertainty. These results show that $\mathrm{CAH}$, like $\mathrm{CSH}$, is not affected by the attack for the duration of our experiment.

We wish to finish this section about the chemical reactions during the attack by mentioning that we have omitted a fundamental aspect of the reaction. Indeed the heterogeneous and evolving nature of the reactive sur- 
face has been observed to be highly influential on the reaction kinetics of minerals [17]. But as nothing has been measured yet in hardened cement attacked by acid concerning the possible disparities of reaction kinetics of individual components, we have considered a constant and uniform reactive surface area, which has enabled to fit perfectly the experimental concentration curves.

\section{Validation of the holographic procedure}

As measurements of pure reaction rates had not been performed up to now, the comparison of our values with other ones, in order to validate them, is not possible for the moment. Indeed, existing studies usually present overall matter loss, including dissolution, precipitation and mass transfer in the solution and inside the porous solid. Therefore the comparison with material loss by pure dissolution is not directly possible. But to enable a future possible link with standard studies of acidic attack of cement-based materials, we have measured the mass loss of our samples during the experiments. To do so, we have dried the attacked platelets at $100^{\circ} \mathrm{C}$ during $2 \mathrm{~h}$, then weighed them. From this and their initial mass, we have computed their mass loss due to the acid attack. Beside, we have estimated this mass loss from the numerical integration of the holographic concentration curve at the last investigated time (just preceding precipitation). As seen in figure 9, the measured and computed values of the mass loss compare well, thus validating the holographic procedure. This check attests that the total quantity of damaged material can then be deduced from the holographic measurements.

To further assess the accuracy of the holographic method, we have also compared the evolution of the quantity of dissolved calcium during the attack, as measured by ICPAES, with the evolution deduced from the numerical integration of the concentration curves measured by holointerferometry, considering that only Portlandite dissolves. As seen in figure 10, both evolutions compare well, the holographic measurements reflect quite quantitatively the progressive release of ions in the solution.

These two agreements between holography and standard measurements validate our methodology and our measured reaction rate constants.

\section{Holographic results}

Figure 11 displays the evolution of the solubility limit of hardened Portland cement in a pure aqueous solution of nitric acid for various $\mathrm{pH}$ obtained by holointerferometry. The quantities of matter are expressed as moles of 


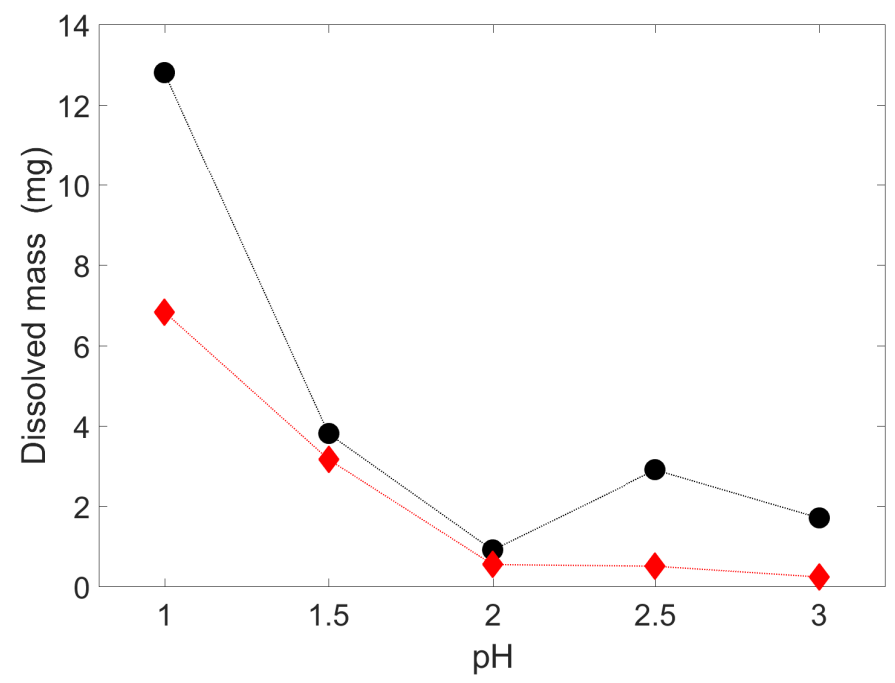

Figure 9: Mass of hydrated cement dissolved in nitric acid at the end the holographic experiment as a function of $\mathrm{pH}$ : measured mass (black circles) and estimated mass from the holographic measurements (red diamonds).

Portlandite (molar mass $M=74 \mathrm{~g} / \mathrm{mol}$ ), the latter being the only constituent contributing significantly to dissolution (section 3 ).

As our measurements are nonstandard ones, we first detail the signification of the found solubilities. If Portlandite is considered to be in excess, the saturation calcium concentration and $\mathrm{pH}$ should be fixed by the solubility product $K_{\mathrm{s}}$ of the reaction $\mathrm{Ca}(\mathrm{OH})_{2} \longrightarrow \mathrm{Ca}^{2+}+2 \mathrm{OH}^{-}$. As $K_{\mathrm{s}}=\left[\mathrm{Ca}^{2+}\right]_{\mathrm{sat}}\left[\mathrm{OH}^{-}\right]_{\text {sat }}^{2}=10^{-5.435}$ and the reaction releases at the same time one calcium and two hydroxide ions, the saturation calcium concentration is $s=\left[\mathrm{Ca}^{2+}\right]_{\mathrm{sat}}=\left(K_{\mathrm{s}} / 4\right)^{1 / 3}=10 \mathrm{mmol} / \mathrm{L}$ and the final $\mathrm{pH}$ is 12.3 . We have added this solubility of calcium in figure 11 as a dashed line. We see that, from $\mathrm{pH} 2$ on, the saturation concentration of the hardened cement corresponds to the one of Portlandite in the case where the $\mathrm{pH}$ is freely fixed by the reaction equilibrium.

We want to stress on the fact that this solubility $s$ is the saturation concentration of calcium in equilibrium exclusively with solid Portlandite, and does not take into account the solubility of other salts precipitating later in the experiment. Subsequently nitrate salts precipitate, and the solubility of calcium is modified accordingly, being in equilibrium with at least two 


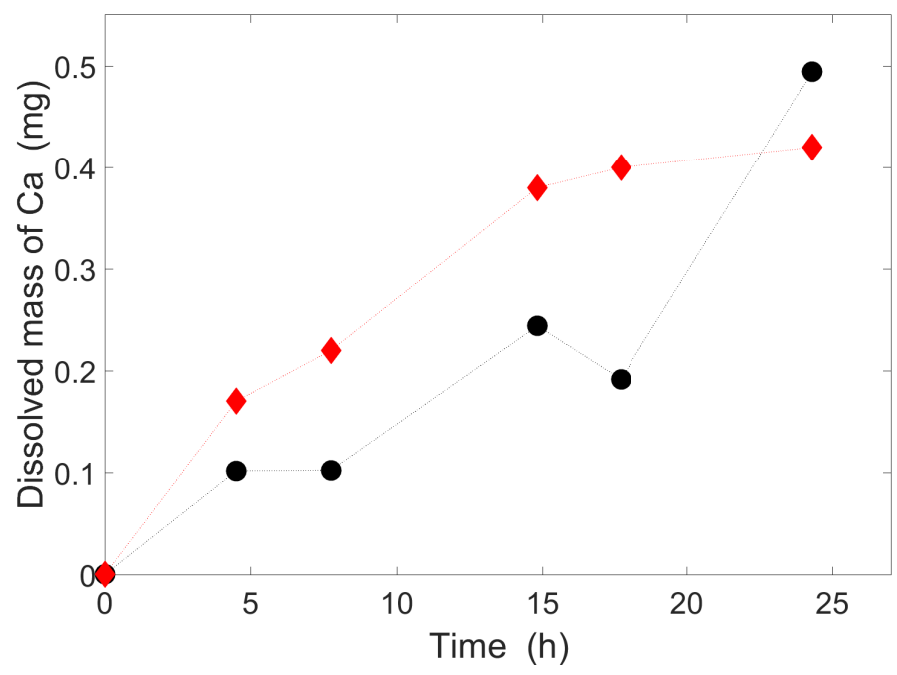

Figure 10: Time evolution of the dissolved calcium mass measured by ICPAES (black circles) and estimated from the holographic measurements (red diamonds) during the attack of hardened cement by nitric acid at $\mathrm{pH}=2$.

solid components (Portlandite and a nitrate salt). For instance in figure 4 the concentrations after $5 \mathrm{~h}$, the time where the calcium concentration starts to decrease due to precipitation, are excluded from the computation of $s$, which is thereby only characteristic of the Portlandite/solution chemical equilibrium.

Two inferences may be drawn from the nature of the $s(\mathrm{pH})$ curve in figure 11. First this is a new corroboration of the fact that Portlandite is here the only dissolving component. Secondly it shows that the $\mathrm{pH}$ of the solution at the interface is not the bulk solution $\mathrm{pH}$, but a $\mathrm{pH}$ fixed by the reaction equilibrium, i.e., $\mathrm{pH}_{\text {surf }}=14+\frac{1}{2} \log \left(K_{\mathrm{s}} / s\right)$. This surface $\mathrm{pH}$ has been added in figure 11. It is observed to be always very alkaline, higher than 11 , even for a bulk solution $\mathrm{pH}$ as low as 1 . The interface $\mathrm{pH}$ takes the equilibrium value fixed by the reaction (12.3) for a bulk $\mathrm{pH} \geq 2$. Below this value, the dissolution kinetics is too fast, compared to the diffusion kinetics, for the equilibrium value to be kept. Whereas the modification of the $\mathrm{pH}$ of the acidic solution in contact with the solid by the cement dissolution was expected, the novelty lies in the fact that this $\mathrm{pH}$ can be accurately measured, which enables a precise knowledge of the condition of 


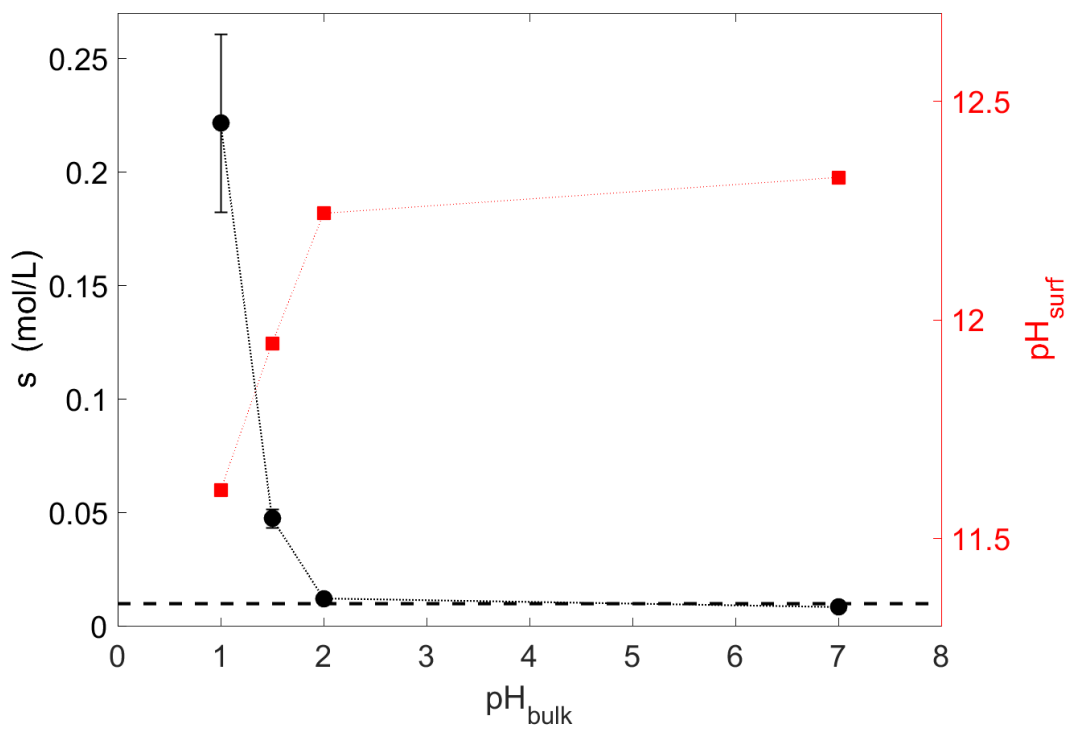

Figure 11: Evolution of the saturation concentration of hardened Portland cement in aqueous solutions of pure nitric acid (black circles) and evolution of the $\mathrm{pH}$ at the interface (red squares) with the $\mathrm{pH}$ of the bulk solution. The values of concentration are in moles of Portlandite per Liter of solution. The dashed line is the saturation concentration of Portlandite in the case that it freely fixes the final $\mathrm{pH}$. 


\begin{tabular}{c|cc|cc}
\multirow{2}{*}{ Acid } & \multicolumn{2}{|c|}{$k$} & \multicolumn{2}{c}{$s$} \\
\cline { 2 - 5 } & $\left(\mathrm{mg} / \mathrm{m}^{2} / \mathrm{s}\right)$ & $\left(\mathrm{mmol} / \mathrm{m}^{2} / \mathrm{s}\right)$ & $(\mathrm{g} / \mathrm{L})$ & $(\mathrm{mol} / \mathrm{L})$ \\
\hline Hydrochloric & 2.6 & 0.035 & 0.9 & 0.012 \\
Nitric & 2.0 & 0.027 & 0.9 & 0.012 \\
Sulfuric & 3.1 & 0.042 & 1.0 & 0.014
\end{tabular}

Table 2: Pure surface reaction rate constant $k$ and solubility of the dissolved hardened cement $s$ for the dissolution of hardened Portland cement in aqueous solutions of various acids for $\mathrm{pH}=2$. Values are expressed both in mass of dissolved hardened cement and in moles of dissolved Portlandite.

the damaging of the cementitious material surface.

Figure 12 (top) displays the evolution of the dissolution rate constant of hardened Portland cement in nitric acid as a function of the $\mathrm{pH}$ of the bulk solution. Again the quantity of matter is expressed in moles of Portlandite. The bulk $\mathrm{pH}$ is the $\mathrm{pH}$ of the initial acidic solution, far from the solidliquid interface. But as has been evidenced in figure 11, the $\mathrm{pH}$ close to the interface has been thoroughly modified by the fast release of the $\mathrm{OH}^{-}$due to the reaction. Therefore paradoxically these reaction rate constants are in fact characteristic of the behavior of the material in very alkaline conditions. We have related in figure 12 (bottom) the rate constants to the true $\mathrm{pH}$ they are characteristic of.

These results may appear puzzling. Indeed to predict the behavior of a cementitious material during an acidic attack at $\mathrm{pH} 1$, the value of the reaction rate constant that should be used is the value at $\mathrm{pH}$ 11.5. This is due to the fact that for a heterogeneous reaction, a mass transport boundary layer develops in the vicinity of the solid. When the dissolution flux and the diffusion flux in this layer are of the same order of magnitude (the mixed kinetics regime), both interact and the concentration and the $\mathrm{pH}$ at the interface take out of equilibrium values (see a detailed situation in [18]).

Table 2 displays the reaction rate constant and saturation concentration of hardened Portland cement in pure aqueous solutions of nitric, sulfuric, and hydrochloric acids at $\mathrm{pH}=2$. We have expressed the quantities of matter in moles of Portlandite and in grams of hardened cement. As for nitric acid, the solubility of hardened cement in sulfuric and hydrochloric acid corresponds to the solubility of Portlandite free to fix the $\mathrm{pH}$. Therefore the $\mathrm{pH}$ of the solution close to the surface for these two acids is likely to be also strongly alkaline, as in figure 11 .

We see that the behavior of the material is similar in the three acid 

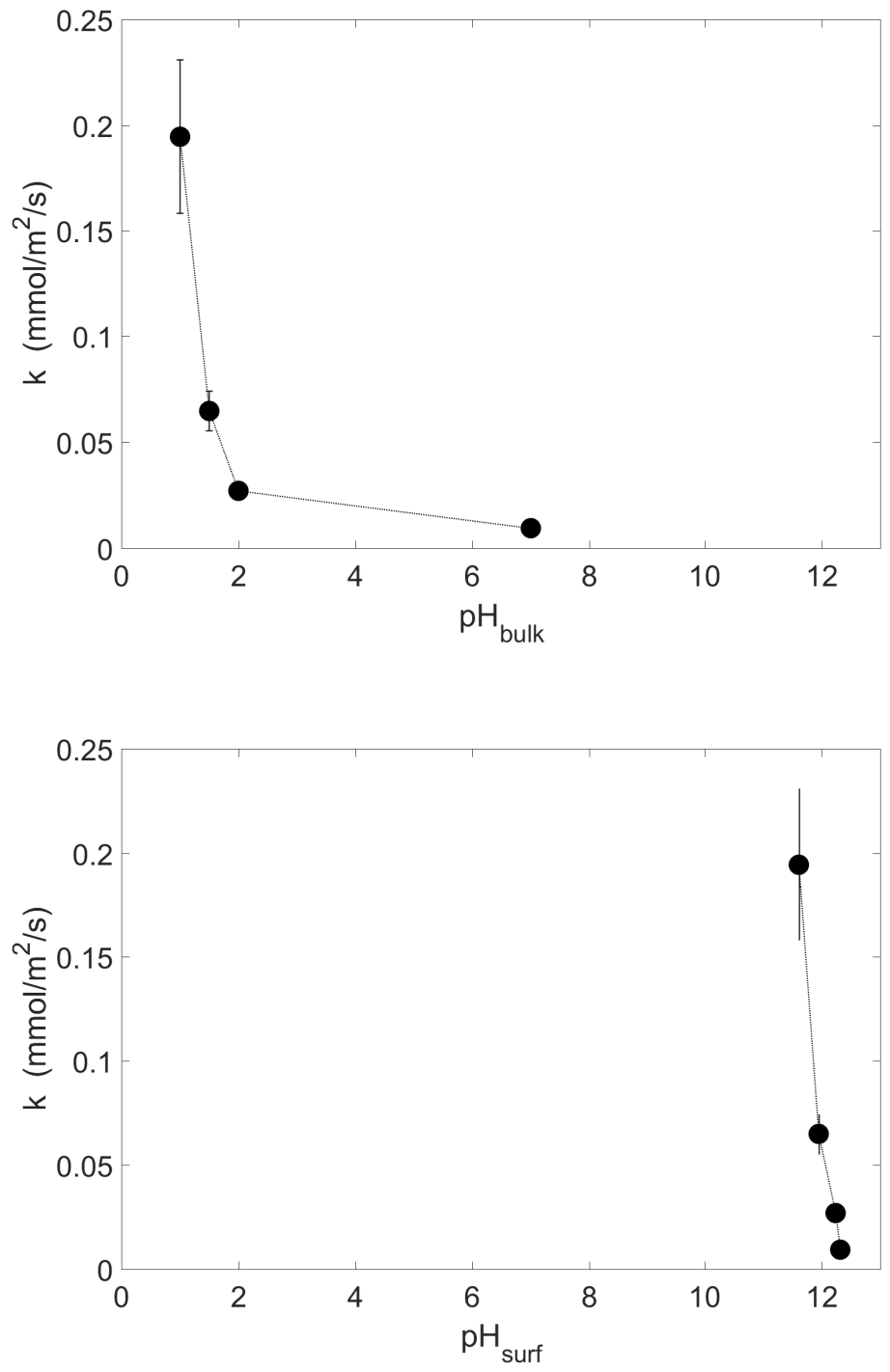

Figure 12: Evolution of the dissolution rate constant of hardened Portland cement in aqueous solutions of pure nitric acid with the bulk $\mathrm{pH}$ of the solution (top) and with the surface $\mathrm{pH}$ deduced from the local solubility (bottom). The values are in millimoles of Portlandite per unit surface and time. 
solutions, with values of $k$ ranging within $25 \%$ from each other. We would like to emphasize that the fact that the reaction rate constants are highly similar for the three investigated acids is opposite to what is expected from conventional studies $[10,2]$. This result demonstrates thus firmly that the kinetics of acid corrosion originates mainly in the precipitation step of the reaction. At this stage, a more or less protective layer progressively covers the surface, modifying the ion detachment rate, whereas before the formation of this layer, the kinetics of all acids are comparable.

Finally, we would like also to recall that our method enables to perform a real-time measurement of the spatially resolved reacting system. Therefore, we have been able to discriminate temporally the dissolution from the precipitation. As seen in figure 4, the time before precipitation sets in, i.e., the time before concentration decreases, is roughly $5 \mathrm{~h}$. This order of magnitude has been observed in all our experiments. It gives an idea of the required time before precipitation proceeds in an unstirred acid. It is linked to the time needed for the interface concentration to reach the solubility of the precipitating salts.

\section{Implications for hardened cement resistance to acid}

As above-recalled, a diffusional boundary layer always builds up over a dissolving solid. In the case of a flowing liquid, this layer shows a thickness of the order of tens of $\mu \mathrm{m}$ [18]. In a quiet liquid, the final boundary layer thickness is theoretically infinite. The interface concentration and $\mathrm{pH}$ depend on this thickness: the $\mathrm{pH}$ will always remain close to 12 , and the concentration will range between 0 and $s(\mathrm{pH})$, for instance $0.22 \mathrm{~mol} / \mathrm{L}$ for $\mathrm{pH} 1$, depending on the relative kinetics of dissolution and diffusion. In the case of acid rain, once the material is wet, it is exposed to a quiescent solution and we are close to the hydrodynamic situation of our experiments. The only difference lies in the geometry, the degradation occurring at the surface and inside the pores, with sizes much smaller than the one of our cell.

Contexts in the laboratory and in real buildings being similar, what can we learn from our experiments for future studies? We propose three investigation directions:

- First, we dispose now of a tool to access the reaction rate of hardened cement in acid. So beside the study of protective layers precipitating after the dissolution step (ettringite ...), the formulation of materials with low reaction rates can also help to manufacture more durable 
concrete. Recent measurements of the dissolution rate of hardened cement at alkaline $\mathrm{pH}$ show that the $\mathrm{Ca} / \mathrm{Si}$ ratio can be optimized to lower this rate. This ratio is likely to influence also the kinetics of acidic attack [19].

- Numerical models of the degradation of concrete in acidic solution make use of empirical laws for the simulation of the dissolution step, rigorous thermochemical parameters being usually not accessible. We have shown here that a nonstandard method can give access to the exact reaction kinetics and we think that the integration of such experimentally-verified laws inside the models can bring a more realistic representation of damaging situations. We would like to emphasize that, even when precipitation sets in, the dissolution rate constants that we have measured before this precipitation, are still valid, being thermodynamically characteristic of the kinetics of the dissolution.

- At last, for industrial situations with harsh acid conditions, where the material is for instance exposed to a long-lasting acidic attack, cementbased materials have to be adapted to this stringent environment. In this case, the introduction in the formulation of admixtures known for their strong adsorption at the hardened cement surface, related to their calcium-complexing nature, could help lowering the dissolution rate of Portlandite in acid. Moreover it is likely that already used admixtures, like superplasticizers, play such a role in adsorbing at the $\mathrm{Ca}(\mathrm{OH})_{2}$ surface. The optimization of their conformation, with the aim of reducing $k$ may lead to more durable materials.

\section{Conclusion}

We have introduced in this article a methodology, including holographic interferometry, that gives access to the space and time resolution of heterogeneous reactions. In the case of the study of the degradation of hydrated cement, it enables to clearly discriminate between the dissolution and precipitation stages of the deterioration. This technique has been used to measure the dissolution rate constant of hardened Portland cement in pure aqueous solutions of hydrochloric, nitric and sulfuric acids at $\mathrm{pH} 2$. Unexpectedly, the three values have been found to compare well, about $1 \mathrm{mg} / \mathrm{m}^{2} / \mathrm{s}$. This is a direct demonstration that the degradation kinetics is mostly driven by precipitation. We have also measured the evolution of the reaction rate constant in nitric acid with the $\mathrm{pH}$. Profiting from the resolving power of the technique, we have finally measured accurately the $\mathrm{pH}$ of the solution 
close to the surface, where it has been found to be always strongly alkaline (between 11.5 and 12.5), whatever the bulk pH. Now that ordinary Portland cement has been studied, this technique may be used to test thoroughly the resistance to acid attack of other cementitious materials, known to offer better acid resistance, like calcium sulfoaluminate cements, or Portland cement containing silica fume.

\section{Acknowledgements}

We thank Maurizio Bellotto, Cédric Desroches, Fernand Chassagneux and Susan Stipp for fruitful discussions.

\section{References}

[1] V. Zivica, A. Bajza, Acidic attack of cement based materials - a review. Part 1. Principle of acidic attack, Construction and Building Materials 15 (2001) 331.

[2] V. Zivica, A. Bajza, Acidic attack of cement based materials - a review. Part 2. Factors of rate of acidic attack and protective measures, Construction and Building Materials 16 (2002) 215.

[3] V. Zivica, Acidic attack of cement based materials - a review. Part 3. Research and test methods, Construction and Building Materials 18 (2004) 683.

[4] F. Girardi, W. Vaona, R. DiMaggio, Resistance of different types of concretes to cyclic sulfuric acid and sodium sulfate attack, Cement \& Concrete Composites 32 (2010) 595.

[5] J. Monteny, N. DeBelie, L. Taerwe, Resistance of different types of concrete mixtures to sulfuric acid, Materials and Structures 36 (2003) 242 .

[6] D. Derry, M. Malati, J. Pullen, Effects of atmospheric acids on Portland cements, Journal of Chemical Technology and Biotechnology 76 (2001) 1049 .

[7] H. Okochi, H. Kameda, S. Hasegawa, N. Saito, K. Kubota, M. Igawa, Deterioration of concrete structures by acid deposition - an assessment of the role of rainwater on deterioration by laboratory and field exposure experiments using mortar specimens, Atmospheric Environment 34 (2000) 2937. 
[8] S. Xie, L. Qi, D. Zhou, Investigation of the effects of acid rain on the deterioration of cement concrete using accelerated tests established in laboratory, Atmospheric Environment 38 (2004) 4457.

[9] M. Chen, K. Wang, L. Xie, Deterioration mechanism of cementitious materials under acid rain attack, Engineering Failure Analysis 27 (2013) 272 .

[10] S. Miyamoto, H. Minagawa, M. Hisada, Deterioration rate of hardened cement caused by high concentrated mixed acid attack, Construction and Building Materials 67 (2014) 47.

[11] R. Beddoe, H. Dorner, Modelling acid attack on concrete: Part i. The essential mechanisms, Cement and Concrete Research 35 (2005) 2333.

[12] V. Zivica, Experimental principles in the research of chemical resistance of cement based materials, Construction and Building Materials 12 (1998) 365 .

[13] J. Colombani, J. Bert, Holographic interferometry for the study of liquids, J. Molec. Liquids 134 (2007) 8.

[14] J. Colombani, J. Bert, Holographic interferometry study of the dissolution and diffusion of gypsum in water, Geochim. Cosmochim. Acta 71 (2007) 1913.

[15] E. Pachon-Rodriguez, J. Colombani, Pure dissolution kinetics of anhydrite and gypsum in inhibiting aqueous salt solutions, AIChE J. 59 (2012) 1622.

[16] J. Colombani, J. Bert, Holographic convection visualization during thermotransport studies - Application to microgravity experiments, Meas. Sci. Technol. 10 (1999) 886.

[17] C. Fischer, R. Arvidson, A. Luttge, How predictable are dissolution rates of crystalline material?, Geochim. Cosmochim. Acta 98 (2012) 177.

[18] J. Colombani, Measurement of the pure dissolution rate constant of a mineral in water, Geochim. Cosmochim. Acta 72 (2008) 5634.

[19] I. Pignatelli, A. Kumar, R. Alizadeh, Y. LePape, , M. Bauchy, G. Sant, Does a dissolution-precipitation mechanism explain concrete creep in moist environments?, arXiv cond-mat (2015) 1508.07082. 